\title{
Understanding Slip Perception of Soft Fingertips by Modeling and Simulating Stick-Slip Phenomenon
}

\author{
Van Anh Ho and Shinichi Hirai \\ Department of Robotics, Ritsumeikan University \\ 1-1-1, Noji Higashi, Kusatsu, Shiga, 525-8577 Japan \\ gr048071, hiraided.ritsumei.ac.jp
}

\begin{abstract}
Slip, especially incipient slip, is a complicated process for soft fingertips; and detection of this slip is important for stable manipulations by both human and robotic fingertips. Experimental research has attempted to explain this phenomenon, but dynamic changes during this process could not be fully delineated. We propose here a dynamic model to investigate the sliding motion of soft fingertips on a plane with friction. The fingertip is comprised of a finite number of elastic, compressible and bendable cantilevers whose free ends act as infinitesimal contact points. The contact surface is afterward meshed using a finite element method based on the coordinates of the contact points. By introducing Coulomb's law and contact compliance into each contact point, we were able to assess the frictional characteristics of the sliding motions of the fingertips. We also could successfully describe the dynamically localized displacements on the contact surface during stick-slip transition, displacements that represent the sliding motion of a soft fingertip. This model can be applied to different shapes of robotic fingertip, including the cylindrical and hemispherical models tested here. We also performed experiments to validate the proposed simulation, including force/moment and vision setups.
\end{abstract}

\section{INTRODUCTION}

Recent research in robotics has focused on the dexterous manipulation of objects using soft fingered robotic hands, especially anthropomorphic hands. This type of research can be categorized into two main groups. The first consists of studies that focus on analyzing the contact mechanics between soft fingers and objects [1]. In the second, tactile sensing systems imitating those of humans, along with many types of sensors, have been developed to simulate human abilities in object grasping and dexterous handling [2]. Whereas the former studies consisted primarily of analysis of stable grasping or object postures controlled by soft fingertips during a pushing or rolling motion on the surface of objects; the latter studies concentrated on the tactile texture perceptions of sensory fingertips, to increase efficiency during object manipulation processes. Among the various movements of fingertips during object handling is slide/slip, which often occurs during contact and is considered important in dexterous manipulation [3]. For example, to assess the texture of an object's surface, the fingertip needs to slide slightly on the surface to extract information about its roughness or friction. The sliding motion of an object between fingertips during grasping, or incipient slip, is a crucial factor in stable object manipulation. However, while the latter studies have addressed all types of fingertip motions, including pressing, rolling, and rubbing; the former studies have focused primarily on pure pushing and rolling movements, while ignoring slip or slide. The difficulties in modeling the sliding motion of fingertips comes from their compliance, their partial movements on contact surfaces, and the force/moment of friction. Kao and Cutkosky [4] have proposed a method that combines compliance and friction on a limited surface to compute the relative sliding motion between a grasped object and soft fingers. That article showed concrete results in modeling contact and in approximation of gross-motion planning. Fearing [5] introduced an algorithm for automatic stable grasping of polygonal objects by two fingers and a point contact with friction. Research has also addressed pushing operations using manipulators. Yoshikawa et al. [6] proposed a pushing method to identify the center of friction of an object with an unknown distribution of friction. In a similar method [7], relevant friction parameters were estimated by performing experimental pushes and observing the resultant motion. Nevertheless, most previous research has dealt only with quasi-static analysis, including gross sliding, not on stick-slip phases (i.e. how and when it occurs), or partial slip on the contact surface of a soft fingertip. In contrast, some contact models have been used in simulation, such as analytical ([8]) and conventional penalty methods ([9]). Although the former methods result in a fast and accurate description of contact, they limit penetration between objects; in contrast, the latter methods can identify the contact area, but can only use penetrative contacts. At the same time, the Finite Element Analysis (FEA) model of a contacting soft fingertip was also developed. This method, however, is time-consuming and usually solved in a static field ([10]). In contrast to the above approaches, Barbagli et al [11] attempted to determine the model that best fit the real rotational friction properties of human fingertips and then extended the object/proxy algorithm to simulate soft finger contact.

In this paper, we have attempted to determine the frictional characteristics of a 3-D soft fingertip during unilateral sliding motion relative to an object. We have proposed a method to model vertical and horizontal deformations of the soft fingertip during sliding by introducing virtual cantilevers. Moreover, we have modeled the contact surface by employing the FEA method, along with Coulomb's frictional law. This can significantly reduce calculation time, while still assuring the dynamic behavior of the system during stick-slip transition. 


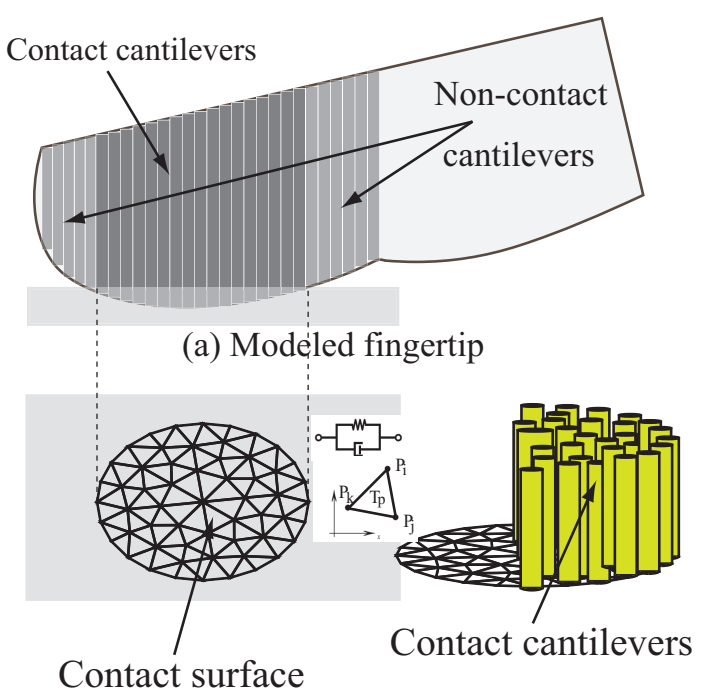

(b) Meshed contact surface

Fig. 1. Model of a sliding soft fingertip with virtual cantilevers and meshed contact surface.

Results from simulation and experimental validation can theoretically explain, for the first time, localized movements on the contact surface during the stick-slip phase, as determined experimentally.

\section{Proposed Hybrid Modeling of A Hemispherical SOFT FINGERTIP}

Many shapes are used to form robotic soft fingertips. We have categorized them into two main groups with respect to grasped objects with rigid flat facets. The first generates uniform distribution of normal stress, such as square and rectangular fingertips. The second is characterized by the nonuniform distribution of contact force, including cylindrical, hemispherical, and human-like fingertips. Both groups are distinguished by rigid fingertips in compliant contact, featuring a pre-slide stage and characteristics of hysteresis. More particularly with the second group, the pre-slide generates localized displacements on the contact surface, which are important in incipient slip detection ([20]). A dynamic model with the appearance of friction is required to represent these phenomena and characteristics of soft fingertips. We have developed a model, which, with some simplifications, can be employed to investigate slip motions of soft fingertips of different shapes, especially during stick-slip transition. Since this model focuses on what happens on the entire contact surface, we have combined discrete and FEA methods to model a general 3-D soft fingertip. By so doing, the calculation times will be reduced considerably, while still assuring the correctness of the model.

Assume that a three dimensional (3-D) soft fingertip with an arbitrary but continuous outer surface is pushed vertically with a specific contact penetration by a normal force $F_{n}$ on a rigid flat plane, and slides horizontally with an external force $F_{t}$ (Fig. 1(a)). Inoue ([1]) proposed a soft fingertip model comprised of an infinite number of vertical elastic virtual springs that could be used to investigate the deformation of the fingertip during pushing or rolling motion on an object. This model, however, was not sufficient to demonstrate sliding motion with the appearance of frictional force. Therefore, instead of virtual springs, we have proposed a model, in which the soft fingertip is comprised of a finite number of virtual elastic cantilevers that are compressible, tensile, and bendable (Fig. 1(b)). This model can represent the diverse deformations of a soft fingertip during a sliding motion, in which the fingertip is pushed and slid at the same time. These cantilevers are fixed on the flat surface of the fingertip, with their free ends on its outer surface. Each cantilever has a uniform circular cross sectional area, whereas the lengths of the cantilevers differ depending on their coordinates within the fingertip. When the fingertip is pushed, some cantilevers will make contact with the object, causing the deformations of these cantilevers. Based on geometrical distributions, we were able to calculate the deformation of each contacting cantilever, which have non-uniform distribution on the contact surface. Afterwards, we meshed the 2-D contact surface using the Voight model to describe the elastic and viscous properties of the contact surface ([12]) on the $\mathrm{O}-X Y$ coordinates. Each node was equivalent to the free end of a contacting cantilever. Each element is a triangle $T_{p}$ with three nodes referred as $P_{i}$, $P_{j}$, and $P_{k}$ in a counterclockwise direction. Fig. 1(c) illustrates a possible mesh of the contact surface. For the sake of simplicity, we made three assumptions:

1) When a cantilever is bent, its deformation is significant only at the free end.

2) Interactions between continuous cantilevers only occur between their free ends on the contact surface.

3) Only cantilevers whose free ends are acting on the contact surface are considered (dark colored cantilevers in Fig. 1(b)). Cantilevers outside the contact surface are deemed irrelevant to the sliding motion of the fingertip (light colored cantilevers in Fig. 1(b)).

As a result, when the fingertip is pushed and slid, its deformation will be represented by deformations of all contacting cantilevers. Moreover, external forces acting on each node on the contact surface can be assessed by calculating the compressing and bending forces of the corresponding cantilever. Especially, the Coulomb friction model can be introduced into each node, a model usually neglected in previous research. Consequently, combining both discrete and FEA methods into a model of a soft fingertip may allow a description of the dynamic behavior of the fingertip during sliding motions, especially in stick-slip states.

\section{MATHEMATICAL MODELS}

\section{A. Cantilever Model}

We ascribe the analysis of one arbitrary cantilever, the $i$ th cantilever, with coordinates in $\mathrm{O}-X Y$ as $\left(x_{i}, y_{i}\right)$, a natural length $l_{o}^{i}$, and natural cross-sectional radius $r_{o}^{i}$. After being pushed with contact depth $d_{n}^{i}$, the normal force $f_{n}^{i}$ acting on 


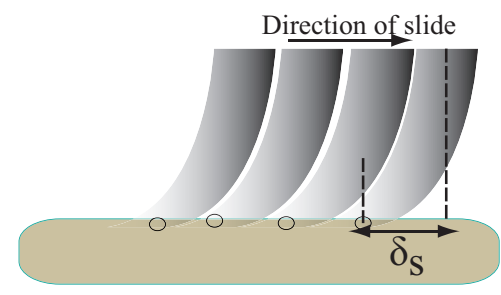

Fig. 2. Bending strain during stick phase.

the free end of this cantilever can be computed as [1]:

$$
f_{n}^{i}=k^{i} d_{n}^{i}=E \frac{\pi r_{o}^{i^{2}}}{l_{o}^{i}} d_{n}^{i},
$$

where $E$ is Young's modulus. Thus, the sum of normal forces acting on all cantilevers is equal to $F_{n}$ :

$$
F_{n}=\sum f_{n}^{i}
$$

When the external tangential force $F_{t}$ is first activated, the fingertip has not been slid yet. The contact surface still sticks to the plane, causing the fingertip to deform. At this time, all contacting cantilevers are bent at their free ends with the same bending strain $\delta s$, as illustrated in Fig. 2. This bending strain is calculated as originated in [15]:

$$
\delta s=\frac{3 \mu F_{n}}{16 R} \frac{2-v}{G}\left\{1-(1-\Phi)^{2 / 3}\right\},
$$

where $\Phi=F_{t} / \mu F_{n}$ is tangential force coefficient, $R$ is the radius of the fingertip, $\mu$ is friction coefficient, $v$ is Poisson's ratio, and $G$ is the shear elasticity's modulus. By assessing the bending strain in Eq. (3), the bending force $f_{b}^{i}$ acting on the free end of the cantilever, with length $l^{i}$ and cross-sectional area's radius $r^{i}$, can be calculated in the following equation:

$$
f_{b}^{i}=b^{i} \delta s=\frac{3 E I}{\left(l^{i}\right)^{3}} \delta s=\frac{3 E \pi\left(r^{i}\right)^{4}}{\left(l^{i}\right)^{3}} \delta s
$$

\section{B. 2-D FEA Modeling of the Contact Surface}

There are many ways to solve FEA simulations of sliding contact using commercial softwares such as ANSYS ${ }^{\mathrm{TM}}$, or MARC $^{\mathrm{TM}}$. These programs, however, cannot determine the exact dynamic behavior of frictional force or of micro slips during stickslip transition. We therefore propose a method to dynamically calculate the output of frictional force, as well as micro sticks/slips on the contact surface during stick-slip transition.

If $\lambda^{\text {ela }}$ and $\mu^{e l a}$ are elastic Lame's constants, $\lambda^{v i s}$ and $\mu^{v i s}$ as viscous Lame's constants, then the connection matrices $\mathbf{J}_{\lambda}$ and $\mathbf{J}_{\mu}$ can be obtained by synthesizing partial connection matrices set of triangles $\left\{T_{p}\right\}$. Detailed derivations of those matrices can be refereed in [16]. Using connection matrices, we can describe a geometric relationship among all nodal points. If $\mathbf{F}_{v e}$ is a visco-elastic force vector on contact nodes, then it can be calculated as:

$$
\mathbf{F}_{v e}=\left(\lambda^{e l a} \mathbf{J}_{\lambda}+\mu^{\text {ela }} \mathbf{J}_{\mu}\right) \mathbf{u}_{N}+\left(\lambda^{v i s} \mathbf{J}_{\lambda}+\mu^{v i s} \mathbf{J}_{\mu}\right) \mathbf{v}_{N},
$$

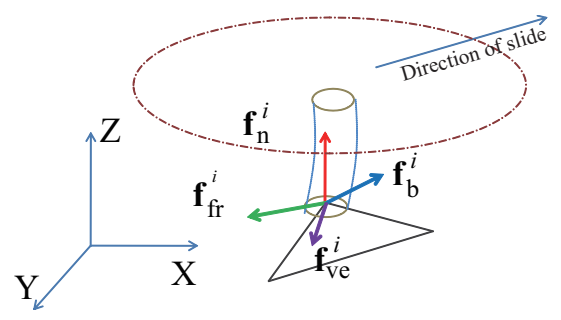

Fig. 3. Analysis of acting force on a arbitrary beam .

or:

$$
\mathbf{F}_{v e}=\mathbf{K}_{\text {ela }} \mathbf{u}_{N}+\mathbf{K}_{v i s} \dot{\mathbf{u}}_{N} .
$$

In addition to the visco-elastic force $\mathbf{f}_{v e}^{i}$ calculated implicitly by Eq. (6), each node has a friction force $\mathbf{f}_{f r}^{i}$, a normal force $\mathbf{f}_{n}^{i}$, and a bending force $\mathbf{f}_{b}^{i}$ (Fig. 3). Friction forces conform to Coulomb's law as they oppose the motion of the contact; thus, if there is no motion at the contact, the friction force can act in any direction with any magnitude less than or equal to the product of the friction coefficient and the normal force. To assess stick/slip motion at each contact node, a slip condition is proposed based on Coulomb condition, and the Constraint Stabilization Method (CSM), which allows the numerical computation of a system of differential equations under geometric constraints ([17]). When applied to a node, CSM assures the fixation of that node, if it keeps sticking to the contact facet. Let $\mathbf{A}_{2,2}^{i}$ be a matrix describing the constraint of the $i$-th node. If $\mathbf{A}^{i}=\mathbf{0}_{2,2}=\mathbf{A}_{0}^{i}$ then this node is unconstrained; while if $\mathbf{A}^{i}=\mathbf{I}_{2,2}=\mathbf{A}_{1}^{i}$ then this node is fixed. As a result, the stick/slip condition of a contacting node can be described as:

$$
\mathbf{f}_{f r}^{i}=\left\{\begin{array}{ll}
\mathbf{f}_{v e}^{i}+\mathbf{f}_{b}^{i} & \text { if } \mathbf{f}_{v e}^{i}+\mathbf{f}_{b}^{i}<\mu \mathbf{f}_{n}^{i} \Rightarrow \text { Stick } \Rightarrow \mathbf{A}^{i}=\mathbf{A}_{1}^{i} \\
\mu \mathbf{f}_{n}^{i} & \text { if } \mathbf{f}_{v e}^{i}+\mathbf{f}_{b}^{i} \geq \mu \mathbf{f}_{n}^{i} \Rightarrow \text { Slip } \Rightarrow \mathbf{A}^{i}=\mathbf{A}_{0}^{i}
\end{array} .\right.
$$

For each iteration, the state of stick or slip of each contacting node can be determined based on the magnitude of the frictional force, allowing the value of the constraint matrix $\mathbf{A}^{i}$ to be determined. The constraint matrix $\mathbf{A}$ of all nodes can be determined by combining partial constraint matrices:

$$
\mathbf{A}^{T}=\left(\begin{array}{cccc}
\mathbf{A}^{0} & \mathbf{0} & \ldots & \mathbf{0} \\
\mathbf{0} & \mathbf{A}^{1} & \ldots & \mathbf{0} \\
\vdots & \vdots & \ddots & \vdots \\
\mathbf{0} & \mathbf{0} & \ldots & \mathbf{A}^{N}
\end{array}\right)_{2 N, 2 N}
$$

where $N$ is the number of contacting nodes. As a result, the geometric constraint can be described in the following equation:

$$
\mathbf{R} \triangleq \mathbf{A}^{T} \mathbf{u}_{N}=\mathbf{0} .
$$

We applied the CSM to incorporate this constraint into dynamic equations. Let us define a critical damping of the constraint:

$$
\ddot{\mathbf{R}}+2 \omega \dot{\mathbf{R}}+\omega^{2} \mathbf{R}=\mathbf{0},
$$




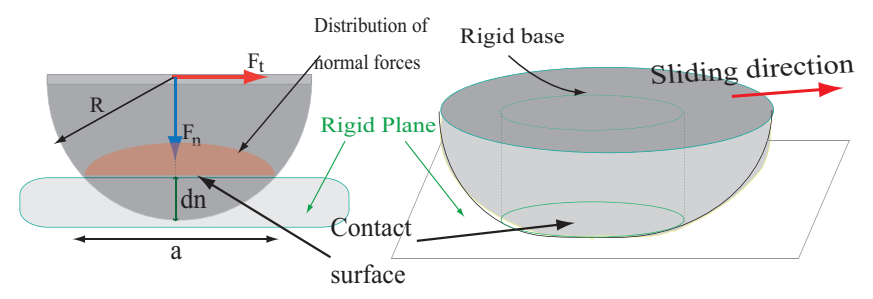

Fig. 4. Model of a hemispherical soft fingertip.

TABLE I

Parameter Values Used in Simulation of Hemispherical FINGERTIP

\begin{tabular}{|c|l|c|}
\hline Parameter & Value & Unit \\
\hline \hline$R$ & 10 & $\mathrm{~mm}$ \\
$d_{n}$ & 2 & $\mathrm{~mm}$ \\
$E$ & 0.623 & $\mathrm{MPa}$ \\
$v$ & 0.48 & \\
$C$ & 100 & Pa.s \\
$\mu$ & 0.9 & \\
\hline
\end{tabular}

where $\omega$ denotes a predetermined angular frequency. This equation turns into the following differential equation:

$$
\mathbf{A}^{T} \ddot{\mathbf{u}}_{N}+\mathbf{A}^{T}\left(2 \omega \dot{\mathbf{u}}_{N}+\omega^{2} \mathbf{u}_{N}\right)=\mathbf{0} .
$$

Consequently, Lagrangian under a set of geometric constraints $\mathbf{R}$ is formulated as:

$$
\mathbf{L}=\mathbf{T}-\mathbf{U}+\mathbf{W}+\lambda^{T} \mathbf{A}^{T} \mathbf{u}
$$

Therefore, a set of motion equations of all nodal points is formulated as:

$$
-\mathbf{M} \ddot{\mathbf{u}}_{N}-\mathbf{K}_{e l a} \mathbf{u}_{N}-\mathbf{K}_{v i s} \dot{\mathbf{u}}_{N}+\mathbf{F}+\mathbf{A} \boldsymbol{\lambda}=\mathbf{0},
$$

where $\mathbf{M}$ is the inertia matrix of the 2-D FEA contact surface ([16]), $\mathbf{F}=\mathbf{F}_{f r}+\mathbf{F}_{b}$ being external force vector on the contact surface, and $\lambda$ being a set of Lagrange multipliers. Recalling the Eq. (11), and introducing the relation $\mathbf{v}_{N}=\dot{\mathbf{u}}_{N}$, equation motions of all nodes can be described as follows:

$$
\left\{\begin{array}{l}
\mathbf{v}_{N}=\dot{\mathbf{u}}_{N} \\
\mathbf{M} \dot{\mathbf{v}}_{N}-\mathbf{A}^{T} \lambda=-\mathbf{K}_{\text {ela }} \mathbf{u}_{N}-\mathbf{K}_{v i s} \mathbf{v}_{N}+\mathbf{F} . \\
-\mathbf{A}^{T} \dot{\mathbf{v}}_{N}=\mathbf{A}^{T}\left(2 \omega \mathbf{v}_{N}+\omega^{2} \mathbf{u}_{N}\right)
\end{array} .\right.
$$

Namely:

$$
\left(\begin{array}{ccc}
\mathbf{I} & \mathbf{0} & \mathbf{0} \\
\mathbf{0} & \mathbf{M} & -\mathbf{A} \\
\mathbf{0} & -\mathbf{A}^{T} & \mathbf{0}
\end{array}\right)\left(\begin{array}{c}
\dot{\mathbf{u}}_{N} \\
\dot{\mathbf{v}}_{N} \\
\lambda
\end{array}\right)=\left(\begin{array}{c}
\mathbf{v}_{N} \\
-\mathbf{K}_{e l a} \mathbf{u}_{N}-\mathbf{K}_{v i s} \mathbf{v}_{N}+\mathbf{F} \\
\mathbf{A}^{T}\left(2 \omega \mathbf{v}_{N}+\omega^{2} \mathbf{u}_{N}\right)
\end{array}\right)
$$

This equation is linear and solvable since the matrix is regular, indicating that we can compute $\dot{\mathbf{u}}_{N}$, and $\dot{\mathbf{v}}_{N}$. Consequently, by combining equations for virtual cantilevers and equations of motion for the contact surface, we can determine the dynamic frictional force acting on each node during its stick/slip motion, as well as the total frictional force on the contact surface. Moreover, we can determine dynamic changes at the contact surface during stick-to-slip phase.

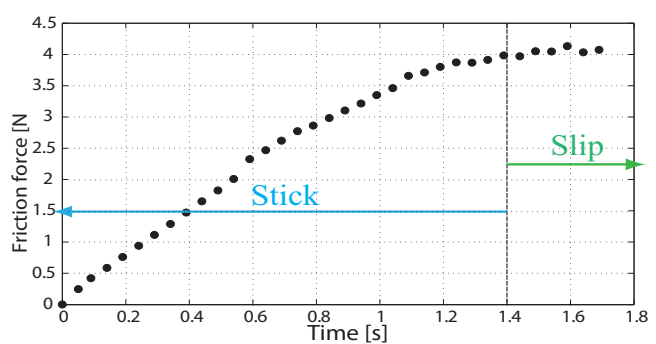

Fig. 5. Friction force during one stick-to-slip transition trial.
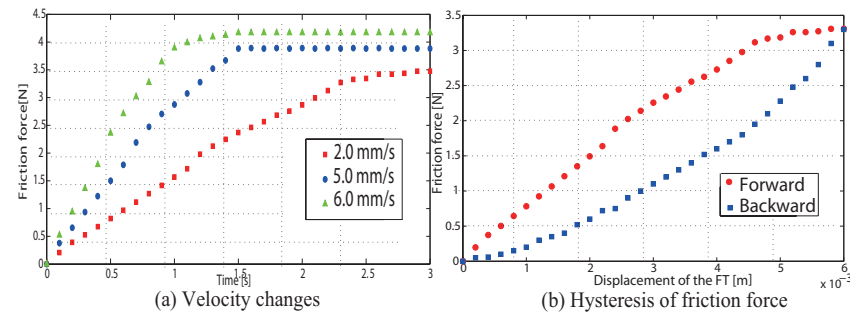

Fig. 6. Responses of friction force of simulation trials.

\section{Simulations}

The simulation was conducted by implementing the model in the $\mathrm{C}++$ programming environment, thus optimizing calculation time. To solve Eq. (14), we employed a sixth order Runge-Kutta method with a sampling time of $1.0 \times 10^{-6} \mathrm{~s}$. The simulation runs on a normal $\mathrm{PC}$ with a $1.8 \mathrm{GHz}$ processor. In this paper, we conducted simulations of two typical soft fingertips, hemispherical and cylindrical fingertips.

\section{A. Hemispherical Soft Fingertip}

In this simulation, the soft fingertip has a diameter of $10.0 \mathrm{~mm}$, similar to the tip of an adult's thumb. The fingertip is pushed with contact depth $d_{n}$ and moved with a constant velocity v. The contact surface is circular with diameter a, which can be calculated as:

$$
a=\sqrt{R^{2}-\left(R-d_{n}\right)^{2}} .
$$

Because the fingertip can slide in any direction, the mesh of the contact surface must be symmetrical to the center of the contact surface. The numbers of contacting nodes and contacting cantilevers were both $N$. The normal force acting on a node on the contact surface can be estimated using a modified form of Eq. (1):

$$
f_{n}^{i}=k^{i} d_{n}^{i}=E \frac{\pi r_{o}^{i^{2}}}{l_{o}^{i}}\left\{\sqrt{R^{2}-\left(x_{i}^{2}+y_{i}^{2}\right)}-\left(r-d_{n}\right)\right\} .
$$

Other parameters are summarized in Table I. Young's modulus $(E)$ and Poisson's ratio $(v)$ have obtained based on linearization of $60 \%$ stress-strain relation of a polyurethane rubber; but the viscous parameter $(C)$ of this type of rubber is not identified. Therefore, we used $100 \mathrm{~Pa} . \mathrm{s}$ as an approximate value for viscous modulus in this simulation. Simulation is focused was stick-to-slip transition, or the transient phase 


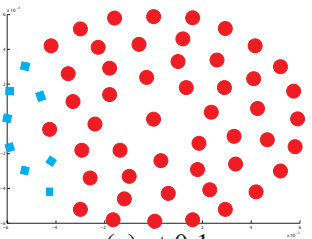

(a) at $0.1 \mathrm{sec}$

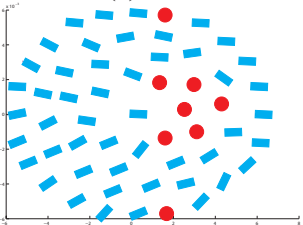

(c) at $1.2 \mathrm{sec}$

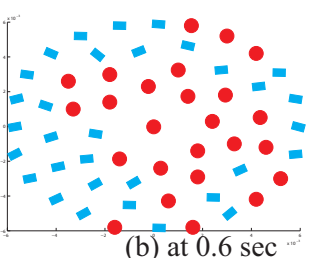

(b) at $0.6 \mathrm{sec}$

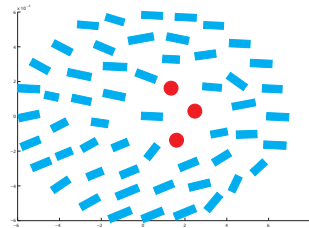

(d) at $1.4 \mathrm{sec}$
Fig. 7. Simulation's micro displacements on the contact surface during stickto-slip transition. Red dots illustrate stick contact nodes, while blue bars show trajectories of slipped contact nodes.

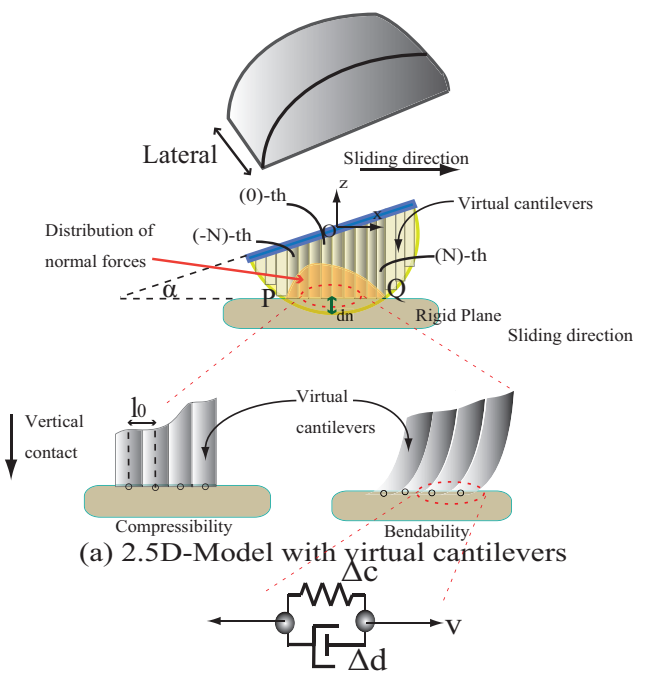

(b) Linkage element on the contact surface

Fig. 8. Simplified model of a sliding cylindrical soft fingertip.

before the stable sliding of the fingertip. By solving Eq. (15), the transient period of stick-slip of the moving hemispherical fingertip can be determined.

Fig. 5 , shows a plot of frictional force acting on the contact surface during the stick-to-slip phase. This force can be calculated by summing all the frictional forces acting on the nodes of the contact surface. Two different stages of friction force can be easily realized during this phase. The friction force keeps increasing during the stick phase, while remaining unchanged in the slide phase. There is no sudden change between the two stages; rather, it occurs smoothly. This phenomenon occurs only with ductile material like that of soft fingertip; but is unrealistic for solid objects when the body accelerates, even if the external forces on the body are below the threshold of the frictional force ([19]). When the sliding velocity varies, the friction responses also change correspondingly (Fig. 6(a)), such that the moment of slide

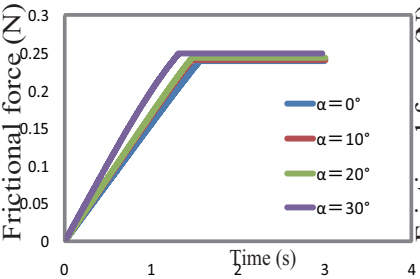

(a) Contact angle changes

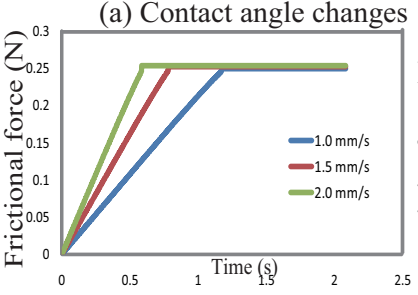

(c) Velocity changes

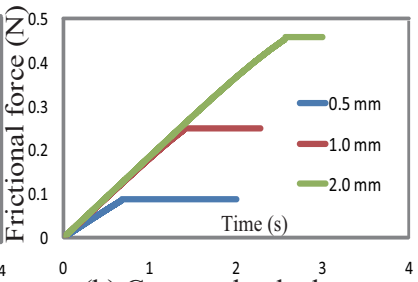

(b) Contact depth changes

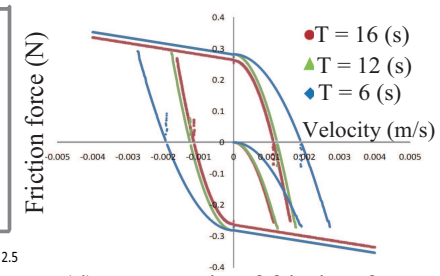

(d) Hysteresis of friction force
Fig. 9. Friction force during stick-to-slip transition when conditions of slide change.

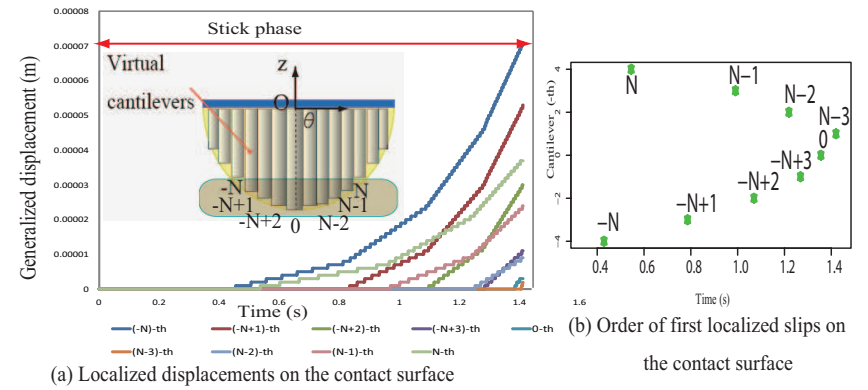

Fig. 10. Localized displacements on the contact surface.

increases as the velocity increases. One of the characteristics of friction of soft material, hysteresis of friction before sliding, can also be perceived in this simulation. The fingertip is moved until the moment right before slide begins, and is then stopped and moved back to its original state. When friction is plotted with regard to displacement of the fingertip (Fig. 6(b)), the forward and backward frictional forces during the stick phase create a hysteresis. The gap of the hysteresis also depends on sliding velocity.

Moreover, by illustrating partial trajectories of contact nodes during the stick-to-slip transition Fig. 7, we can closely look at micro changes of strain on the contact surface. That is, the peripheral areas of the contact surface slip first (Fig. 7(a)), and the slipped areas propagate to the center of the contact surface Fig. 7(b) to (c). This incipient slip of a hemispherical soft fingertip has been described in ([2],[10]). Moreover, during experimental stick-to-slip transition the center of the contact surface was the last to give way right before gross slippage of the moved fingertip on the plane of the object [20].

\section{B. Cylindrical Soft Fingertip}

When a cylindrical fingertip makes contact with a plane, the distribution of normal force is uniform along the lateral dimension (Fig. 8(a)). Therefore, it is not necessary to mesh 

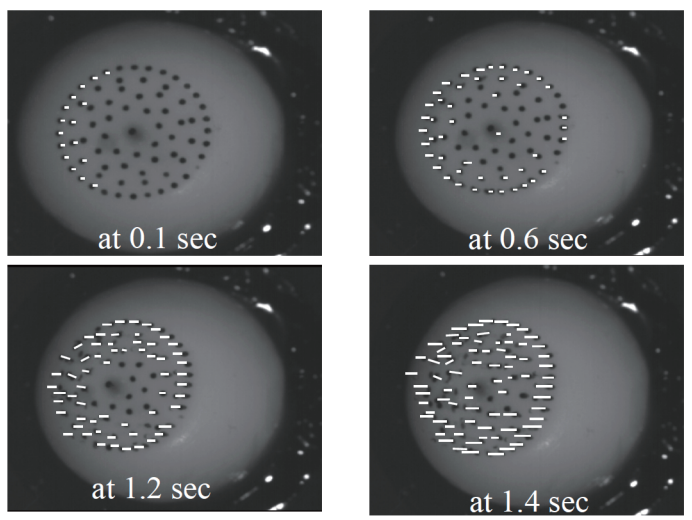

Fig. 11. Experiment's micro displacements on the contact surface during stick-to-slip transition. White bars show trajectories of slipped contact nodes.

the entire contact surface precisely. Rather, the proposed 3-D model in Section II can be simplified to a $2.5-\mathrm{D}$ model by choosing cantilevers with rectangular cross-sectional areas. At this time, the contact surface includes only linkage elements (Fig. 8(b)), corresponding to triangular elements in the 3D model. This can reduce the time required for simulation compared with that needed for hemispherical fingertips; but still assure the correctness of the simulation. Some parameters used for simulation can be found in [13].

Fig. 9(a)-(c) shows that the responses of friction force differ significantly when some conditions of slide change, such as sliding velocity, contact angle, and contact depth, similar to responses occurring for hemispherical fingertips. In particular, we are able to observe the hysteresis of frictional forces upon periodic changes in velocity (Fig. 9(d)). In addition, the hysteresis curves became narrower when the rate of velocity changes increased ([19]). We could also assess the localized displacements of nodes on the contact surface during stick-slip transition. Fig. 10 illustrates the displacements of contacting nodes numbered $-N$ to $N$, from left to right along the direction of slide. The localized slip also propagates from outward to inward, and the zone gives way before gross slip is adjacent to the center zone.

As described above, simulation trials were implemented in a $\mathrm{C}++$ environment, using a common PC. We were able to conduct simulations of the sliding cylindrical fingertip model in real time. Using our model of a hemispherical fingertip, each trial took 4 to 10 minutes, depending on initial conditions such as sliding velocity and contact depth. We are attempting to accelerate simulation trials by utilizing power of parallel computations, using CUDA in GPU-equipped computers. This seems very promising for future implementations of simulation in real time. Compared with solid FEA simulations implemented in commercial software ([14]), the simulation time was markedly reduced. The solid FEA simulation apparently includes detailed stress-strain information at any node, whereas our model can describe stick-slip transitions dynamically with promising application in real time.

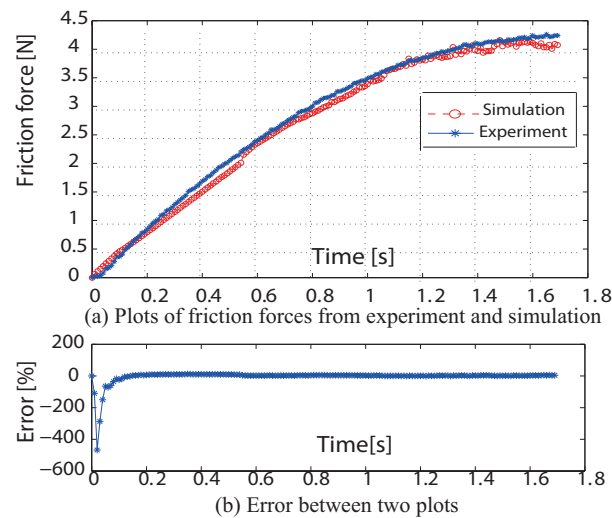

Fig. 12. Comparison between simulation and experiment's friction force at $v=5.0 \mathrm{~mm} / \mathrm{s}$.

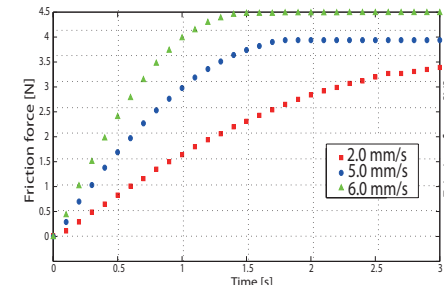

(a) Velocity changes

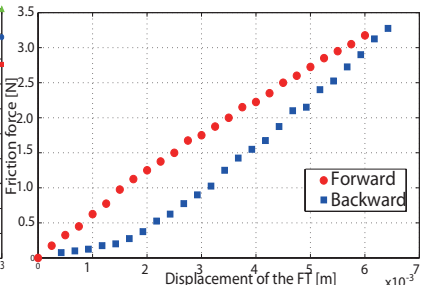

(b) Hysteresis of friction force
Fig. 13. Experimental friction force during stick-to-slip transition.

\section{EXPERIMENTAL VALIDATION}

\section{A. Hemispherical Soft Fingertip}

To verify the simulated model, we conducted an experiment in which one polyurethane rubber soft fingertip was moved on a rigid surface by a 2-DOF stage. A 3-DOF loadcell was attached to the fingertip to measure external forces acting on it. Moreover, the outer surface of the fingertip contains dots representing contact nodes. These dots have coordinates similar to those of contacting nodes in simulation. To create these dots, we utilized a thin steel panel with drilled holes of $0.5 \mathrm{~mm}$ radius distributed similarly to those of nodes on the meshed contact surface. This plate was pushed vertically on the top of the soft fingertip with the same contact depth $d_{n}$ as in the simulation. Spray paint was used to create black dots through the drilled holes (Fig. 11). A high speed camera was employed to track the movements of these dots. The white bars in Fig. 11 illustrate localized displacements of tracked dots on the contact surface during stick phase. We also found that localized slips on the contact surface occurred first at the outward border, later propagating into the center zone. Consequently, this experimental result validates our proposed model and its simulation result.

Fig. 12(a) compares simulated and experimental frictional forces during stick-to-slip transition at $v=5.0 \mathrm{~mm} / \mathrm{s}$. We observed very good agreement between the two results. The relative error was large at the very beginning of the slide, but quickly vanishes (Fig. 12(b)). When we assessed changes in frictional force as a function of the velocity of the slide 


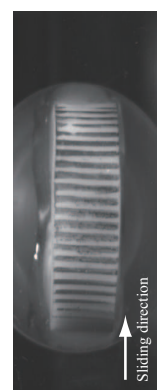

(a)

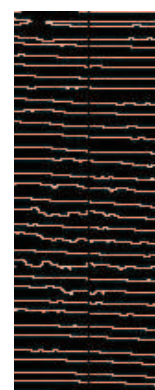

(b)

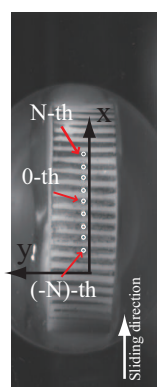

(c)
Fig. 14. Images for processing: (a) Gray-scale image. (b) Binary image with extracted edges. (c) Image with tracked points.

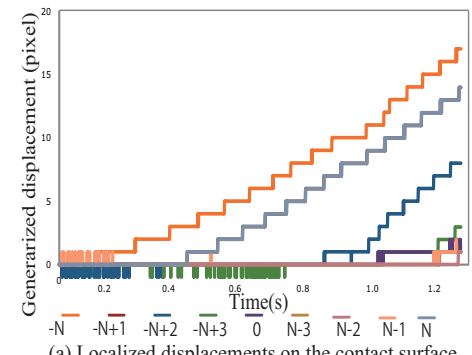

(a) Localized displacements on the contact surface

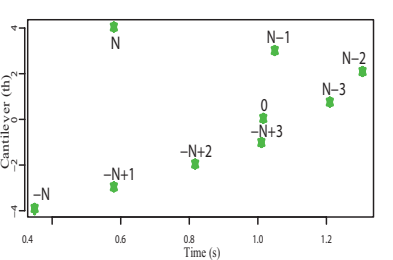

(b) Order of first localized slips on the contact surface
Fig. 15. Localized displacements on the contact surface.

Fig. 13(a), we found that, as velocity increases, the overt slip occurs sooner and the coefficient of friction increases. The latter is due to the viscosity characteristics of frictional forces, in that the coefficient of friction depends on sliding velocity ([12]). This was also observed in the simulation results, as mentioned in Section IV. Hysteresis of friction force relative to fingertip displacement is plotted in Fig. 13(b), showing general agreement with simulation results (Fig. 6(b)).

\section{B. Cylindrical Soft Fingertip}

Experimental apparatuses similar to those for hemispherical fingertip were also employed for validation of cylindrical fingertips. In the visual setup, the outer surface of the fingertip has a distribution of continuous ridges, which were marked for ease of tracking (Fig. 14). We observed movements of the center points of contacting ridges on the contact surface. These center points represent contacting nodes in the model. The trajectories of the contacting nodes are illustrated in Fig. 15, showing agreement with that of simulation result in Fig. 10. The force validation shown in Fig. 16 is also in agreement with the simulation results in Fig. 9. Consequently, our experimental results totally support our proposed simulation model.

\section{DISCUSSION}

\section{A. Stick-Slip Transition}

In this paper, we employed two typical shapes of robotic soft fingertips, hemispherical and cylindrical, to investigate sliding motions using our proposed model. We believe that our
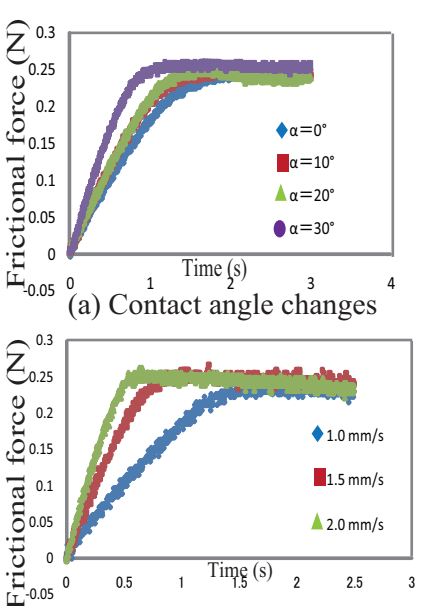

(c) Velocity changes

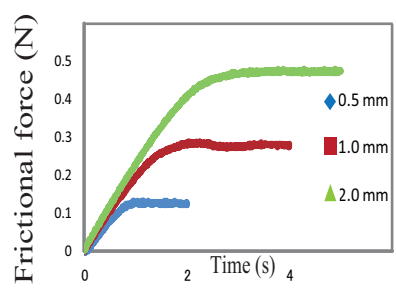

(b) Contact depth changes

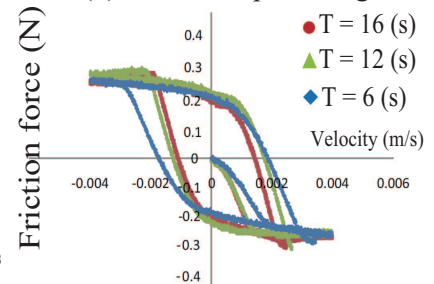

(d) Hysteresis of friction

Fig. 16. Friction force during stick-to-slip transition conditions of slide change.

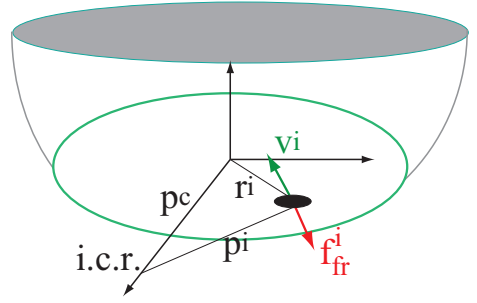

Fig. 17. Contact surface and i.c.r.

model can be used to simulate other types of soft fingertips, even those with complicated surfaces. Unless the distribution of normal forces on the contact surface is impossible to perceive, stick-slip transitions and localized displacements can be assessed using our model. This model can also be used to explain the sliding of human fingertips. This conclusion is supported in part by previous experiments on the sliding motion of human fingertips with epidermal ridges ([21], or [22]) especially on how the contact surfaces of human fingertips change during sliding motion from stick to slip states. Fig. 6 in Ref. [22] is similar to our simulation results; in that localized displacements occur first at the border zones and later propagate inward. As a result, our model promises a platform that can dynamically represent the sliding motion of many soft fingertips, ranging from robotic to human.

For a complex model with various materials, such as a human finger, our group has used magnetic resonance imaging and the Snake algorithm to specify the exact shape, as well as the locations of bone and other tissues. Based on this information, the stiffness of each cantilever corresponding to bone, soft tissue, and other materials can be identified. The physical connection between cantilevers of different stiffness assures the flawlessness of the model. The contact surface is meshed with corresponding characteristics of human skin. Thus, by extending our proposed model, we can simulate the sliding motion of human fingers. 


\section{B. Friction Torsion}

In investigating the bilateral movement of soft fingertips, it was necessary to introduce frictional torque into the model [23]. To analyze this movement, we employed instantaneous center of rotation (i.c.r.), which has been mentioned in [4]. The moment about the $z$-axis, or normal to the contact area, can be calculated as: :

$$
m=-\sum_{i}^{N} m^{i}=-\sum_{i}^{N} \mathbf{r}^{i} \times \frac{\mathbf{v}^{i}}{\left\|\mathbf{v}^{i}\right\|} f_{f r}^{i},
$$

with $f_{f r}^{i}$ be the friction force at the contacting node $i$ calculated in Eq. (7); $\mathbf{v}^{i}$ is the velocity vector with respect o the i.c.r.; and $\mathbf{r}^{i}$ is the location vector of the $i$-th node.

By using this hypothesis, more constraints need to be introduced for all contacting nodes of this model. With respect to i.c.r., for each iteration the direction of movement of each contact node is considered perpendicular to $\mathbf{p}^{i}$, i.e.:

$$
Q^{i}=\mathbf{v}^{i} \cdot \mathbf{p}^{i}=v_{x}^{i} p_{x}^{i}+v_{y}^{i} p_{y}^{i}=0 .
$$

This is a nonholonomic constraint. Let $\mathbf{Q}$ be the matrix description of Eq. (20) for all contact nodes. Since $\mathbf{Q}$ contains velocity components, any differential equation with second order derivatives of $\mathbf{Q}$ is not available. Thus, instead of using Eq. (10), we apply the following differential equation:

$$
\dot{\mathbf{Q}}+v \mathbf{Q}=0,
$$

where $v$ is a predetermined damping ratio. In companion with holonomic constraint described in Eq. (9), we can incorporate these constraints into equations of motion as [24]:

$$
\left(\begin{array}{cccc}
\mathbf{I} & \mathbf{0} & \mathbf{0} & \mathbf{0} \\
\mathbf{0} & \mathbf{M} & -\Phi^{H T} & -\Phi^{N T} \\
\mathbf{0} & -\Phi^{H} & \mathbf{0} & \mathbf{0} \\
\mathbf{0} & -\Phi^{N} & \mathbf{0} & \mathbf{0}
\end{array}\right)\left(\begin{array}{c}
\dot{\mathbf{u}}_{N} \\
\dot{\mathbf{v}}_{N} \\
\lambda^{H} \\
\lambda^{N}
\end{array}\right)=\left(\begin{array}{c}
\mathbf{v}_{N} \\
\boldsymbol{F} \\
\gamma^{H} \\
\gamma^{N}
\end{array}\right)
$$

In this equation, $\Phi^{H}$ and $\Phi^{N}$ are holonomic and nonholonomic constraint matrices calculated from constraints $\mathbf{R}$ and $\mathbf{Q}$ respectively according to [24]; $\lambda^{H}$ and $\lambda^{N}$ are sets of Lagrange multipliers; $\gamma^{H}$ and $\gamma^{N}$ are derived from Eqs. (10) and (20). By numerically integrating these equations, responses of friction force and friction torque can be assessed. We will report this result in another paper soon.

\section{CONCLUSION}

This paper presents a hybrid FEA model of discretely represented virtual cantilevers, which simulate the dynamic behavior of a sliding soft fingertip. We have focused on the stick-slip transition of this slide, which has been considered an important factor in stable grasping. Our simulation successfully reproduced dynamically localized displacements on the contact surface during stick-slip transition, which had been observed experimentally beforehand. Our validation experiments indicate that our model is sufficient to explain dynamically this complex phase of a sliding soft fingertip.

\section{REFERENCES}

[1] T. Inoue, S. Hirai, Elastic Model of Deformable Fingertip for SoftFingered Manipulation, IEEE Transaction on Robotics, vol. 22, no. 6 , December 2006.

[2] L. Beccai, S. Roccella, L. Ascari, P. Vandastri, A. Sieber, M. C. Carrozza, P. Dario, Development and Experimental Analysis of a Soft Compliant Tactile Microsensor for Anthropormorphic Artificial Hand, IEEE Transaction on Mechatronics, vol. 13, no. 13, April 2008.

[3] A. M. Okamura, Micheal L. Turner, and Mark R. Cutkosky, Haptic Exploration of Objects with Rolling and Sliding, IEEE Int. Conf. Robot. Autom., vol. 3, pp. 2485-2490, May 1997.

[4] I. Kao, M. R. Cutkosky, Quasistatic Manipulation with Compliance and Sliding, The Int. Jour. of Robotic Research, Vol. 11, No. 1, pp. 20-40, Feb. 1992.

[5] R. S. Fearing, Implementing a force strategy for object reorientation, IEEE Int. Conf. on Robotics and Autom., vol. 3, pp. 96-102, April 1986.

[6] T. Yoshikawa and M. Kurisu, Identification of the Center of Friction from Pushing an Object by a Mobile Robot, Proc. of IEEE/RSJ Int. Workshop on Intelligent Robot and Systems, pp. 449-454, 1991.

[7] Kevin M. Lynch, Estimating the Friction Parameters of Pushed Objects, Proc. of IEEE/RSJ Int. Conf. on Intelligent Robot and Systems, pp. 186193, July 1993.

[8] D. Baraff, Analytical methods for dynamic simulation of non penetrating rigid bodies, SIGGRAPH Compute Graph, vol. 23, no. 3, pp. 223-232, 1989.

[9] G. Hippmann, An algorithm for compliant contact between complexly shaped bodies, Multibody System Dynamics, vol. 12, no. 4, pp. 345. 362, December 2004.

[10] T. Maeno, S. Hiromitsu, and T. Kawai, Control of Grasping Force by Detecting Stick/Slip Distribution at the Curved Surface of an Elastic Finger, IEEE Int. Conf. on Robotics and Autom., pp. 3895-3900, April 2000.

[11] F. Barbagli, A. Frisoli, K. Salisbury, and M. Bergamasco, Simulating human fingers: a Soft Finger Proxy Model and Algorithm, 12th International Symposium on Haptic Interfaces for Virtual Environment and Teleoperator Systems, , pp/ 9-17, 2004.

[12] J. L. Johnson, Contact Mechanics, Cambridge, U.K., Cambridge Univ. Press, 1984

[13] V. A. Ho, S. Hirai, Modeling, Analysis of a Frictional Sliding Soft Fingertip and Experimental Validations, International Journal of Advanced Robotics, Vol. 26, 2011 (In press).

[14] V. A. Ho, D. V. Dzung, S. Sugiyama, S. Hirai, Analysis of sliding of a Soft Fingertip Embedded with a Novel Micro Force/Moment Sensor: Simulation, Experiment, and Application, 2010 IEEE International Conference on Robotics and Automation, pp 889-894, Kobe, May, 2009.

[15] R. D. Mindlin, Compliance of elastic bodies in contact, Trans. ASME, J. Appl. Mech., vol. 16, pp. 259-268, 1949.

[16] K. Namima, Z. Wang, and S. Hirai, Simulation of Soft Fingertip Deformation under Contact and Rolling Constraints using FEM and CSM, IEEE Int. Conf. on Robotics and Biomimetics (Robio 2009), pp.1585-1590, China, Dec. 19-23, 2009

[17] J.Baumgarte, Stabilization of Constraints and Integrals of Motion in Dynamical Systems, Computer Methods in Applied Mechanics and Engineering, Vol.1, pp.1-16, 1972

[18] C. Canudas, H. Olsson, K. J. Astrom, P. Lischinsky, A New Model for Control of Systems with Friction, IEEE Trans. on Automatic Control, Vol. 40, No. 3, pp. 419-425, March, 1995.

[19] D. A. Haessig, B. Friedland, On the Modeling and Simulation of Friction, in Proc. American Control Conference, pp.1256-1261, 1990.

[20] J. S. Son, E. A. Monteverde, and R. D. Howe, A Tactile Sensor for Localizing Transient Events in Manipulation, Proc. IEEE Int. Conf. on Robotics and Automation, vol. 1, pp. 471-476, May 1994.

[21] Levesque, V. and Hayward, V., Experimental evidence of lateral skin strain during tactile exploration, Proc. 2003 Eurohaptics, pp. 261-275.

[22] M. Tada, T. Kanade, An imaging system of incipient slip for modeling how human perceives slip of a fingertip, Proceedings of the 26th Annual International Conference of the IEEE EMBS, pp. 2045-2048.

[23] Allison M. Okamura, Niels Smaby, and Mark R. Cutkosky An Overview of Dexterous Manipulation, IEEE Int. Conf. Robot. Autom Symposium, May 2000.

[24] J. Jalon, and E. Bayo, Kinetic and Dynamic Simulation of Multibody Systems, Springer Verlag, Chapter 5, 1993. 